\title{
Hypomania in Bobble-Head Doll Syndrome: A Case Report of Surgically Treated Stereotypy and Hypomania
}

\author{
Myong Hun Hahm 1 , Jungmin Woo ${ }^{2}$, and Ki Hong $\mathrm{Kim}^{3}$ \\ ${ }^{1}$ Department of Radiology, School of Medicine, Kyungpook National University, Daegu, Republic of Korea \\ ${ }^{2}$ Department of Psychiatry, School of Medicine, Kyungpook National University, Daegu, Republic of Korea \\ ${ }^{3}$ Department of Neurosurgery, School of Medicine, Catholic University of Daegu, Daegu, Republic of Korea
}

\begin{abstract}
A 22-year-old man was admitted with gradually aggravating stereotypic head movement with hypomania. Brain magnetic resonance imaging showed a large suprasellar arachnoid cyst extending into the third ventricle, with obstructive hydrocephalus, characteristic of bobble-head doll syndrome. Endoscopic fenestration of the suprasellar arachnoid cyst was performed. Stereotypic head movement stopped immediately after surgery and hypomanic symptoms gradually improved within a month. During 4 years of follow-up observation without medication, neuropsychiatric symptoms did not relapse. We report our experience of surgically treating stereotypy and hypomania in a case of bobble-head doll syndrome and discuss the possible neuropsychiatric mechanisms of this rare disease.
\end{abstract}

Psychiatry Investig 2018;15(5):546-549

Key Words Arachnoid cysts, Bobble-head doll syndrome, Mania, Stereotypy.

\section{INTRODUCTION}

Bobble-head doll syndrome (BHDS) is a rare neurological stereotypic head movement disorder characterized by about $2 \mathrm{~Hz}$ to $3 \mathrm{~Hz}$ of periodic anterior-posterior and occasionally side-to-side head movements. ${ }^{1,2}$ Organically, this syndrome is almost always associated with a cystic lesion in or near the third ventricle, causing pressure on periventricular neuronal structures, including the thalami. Previously reported causes include suprasellar arachnoid cyst (AC); third ventricular cyst; cystic choroid plexus papilloma of the third ventricle; cyst in the cavum septum pellucidum; and less commonly, aqueductal stenosis, shunt malfunction, or Dandy-Walker complex malformation. ${ }^{1,3,4}$ The rhythmic head bobbling is aggravated by emotional stress and can be decreased in frequency voluntarily and stops during sleep. The syndrome commonly presents prior to 10 years of age, and surgical treatment is the only treatment method, including cyst decompression by mi-

\footnotetext{
Received: September 5, 2017 Accepted: October 25, 2017

$\bowtie$ Correspondence: Jungmin Woo, MD, PhD

Department of Psychiatry, School of Medicine, Kyungpook National University, 680 Gukchaebosang-ro, Jung-gu, Daegu 41944, Republic of Korea Tel: +82-53-200-5747, Fax: +82-53-426-5361, E-mail: woojm3@hanmail.net (ac) This is an Open Access article distributed under the terms of the Creative Commons Attribution Non-Commercial License (http://creativecommons.org/licenses/bync/4.0) which permits unrestricted non-commercial use, distribution, and reproduction in any medium, provided the original work is properly cited.
}

crosurgical fenestration of the cyst via a transcranial approach or by insertion of a device such as a cysto-peritoneal shunt. ${ }^{1,2,5}$

Here, we report a case of BHDS with hypomania, rarely combined symptoms caused by a large suprasellar AC. We share the experience of surgically treating the patient's stereotypy and mood disorder.

\section{CASE}

A 22-year-old previously healthy man visited the outpatient department of neuropsychiatry in our institution. He had been suffering from involuntary abnormal head movement for 5 months. The movement was characterized by $4-5 \mathrm{~Hz}$ of anterior-posterior head movements, followed by 3-4 Hz of side-to-side movements. This head movement pattern was rhythmic, similar to that seen with a nodding- and shakinghead doll, and worsened under stressful situations. It could, however, be decreased voluntarily, and it disappeared completely during sleep.

He was first diagnosed at a local neuropsychiatric clinic as having a tic disorder and managed for the same; imaging was not performed because the abnormal head movement was stereotypic, as seen in tic disorder, obsessive-compulsive disorder, repetitive behavior, and other stereotypies. However, the movement gradually worsened, and even new psychiatric 
symptoms developed 3 months prior to presentation. The psychiatric symptoms included elated mood, talkativeness, inflated self-esteem, distractibility, and intermittent aggressiveness; these could be clinically diagnosed as hypomania. Initially, hypomania was not clinically significant, and only an observation was made. Finally, however, he was referred to our institution for further management because all symptoms had aggravated over time.

During baseline evaluation after admission, brain magnetic resonance imaging (MRI) revealed a large cystic lesion in the suprasellar region extending into the third ventricle, and hydrocephalus was observed in lateral ventricles (Figure 1). There was no enhancement or solid portion in the thin cyst wall, and the internal signal exhibited by the cystic contents was homogenous and suppressed in the fluid-attenuated inversion recovery sequence. Accordingly, a radiological diagnosis of suprasellar AC was made. The lateral ventricles were severely dilated, and the midbrain and pons were anteriorly compressed by the cyst, although the fourth ventricle was of normal size, indicating obstructive hydrocephalus. Although the optic chiasm, pituitary gland, and pituitary stalk were also severely compressed and displaced, visual disturbance was not observed, and the endocrine profile was normal. Combined with stereotypic head movement, similar to that in a bobble-head doll, these findings were indicative of bobblehead doll syndrome (BHDS).

Neurosurgeons performed endoscopic fenestration of the cyst wall to facilitate flow of cerebrospinal fluid (CSF) to the lateral ventricle and basal cistern. They performed a large fenestration to the lateral ventricle by incision of the cyst wall: an opening to the prepontine cistern by scissoring of the bottom of cyst wall and Liliequist membrane. Consequently, the cyst communicated with the lateral ventricle superiorly and with the prepontine cistern inferiorly. Subsequently, the cyst shrunk.

The patient's stereotypic head movement completely stopped on postoperative day 1 . However, his hypomanic symptoms persisted, and $50 \mathrm{mg} /$ day quetiapine, the atypical antipsychotic drug, was administered because he reported difficulty in controlling his behavior during hospitalization. After drug administration, he could maintain social relationships with surrounding people. Postoperative computed tomographic scanning performed 1 week later revealed a reduction in the size of the lateral ventricle and a decompressed cyst within the third ventricle (Figure 2). A month after surgery, other hypomanic symptoms also improved, and he was discharged with a euthymic status.

After discharge, the same dose of quetiapine was maintained for 2 months and then discontinued. During 4 years of follow-up observation without medication, neuropsychiatric symptoms including stereotypic head movement and hypomania did not relapse.

\section{DISCUSSION}

Almost all patients with BHDS exhibit head movement in the vertical direction, similar to nodding. Unlike that in previous reports, our patient showed a mixed type of stereotypy: anterior-posterior followed by side-to-side head movements and accompanying mood symptoms. To our knowledge, this mixed pattern of BHDS with mood symptoms caused by a suprasellar AC has not previously been reported. ${ }^{6}$

The pathophysiology of BHDS is not clearly understood. Benton et al. ${ }^{7}$ suggested that in BHDS, distortion of the third ventricle by a cystic lesion causes impairment of normal function of the surrounding structures. Russo et al. ${ }^{2}$ reported that the cause of BHDS was extrapyramidal dysfunction due
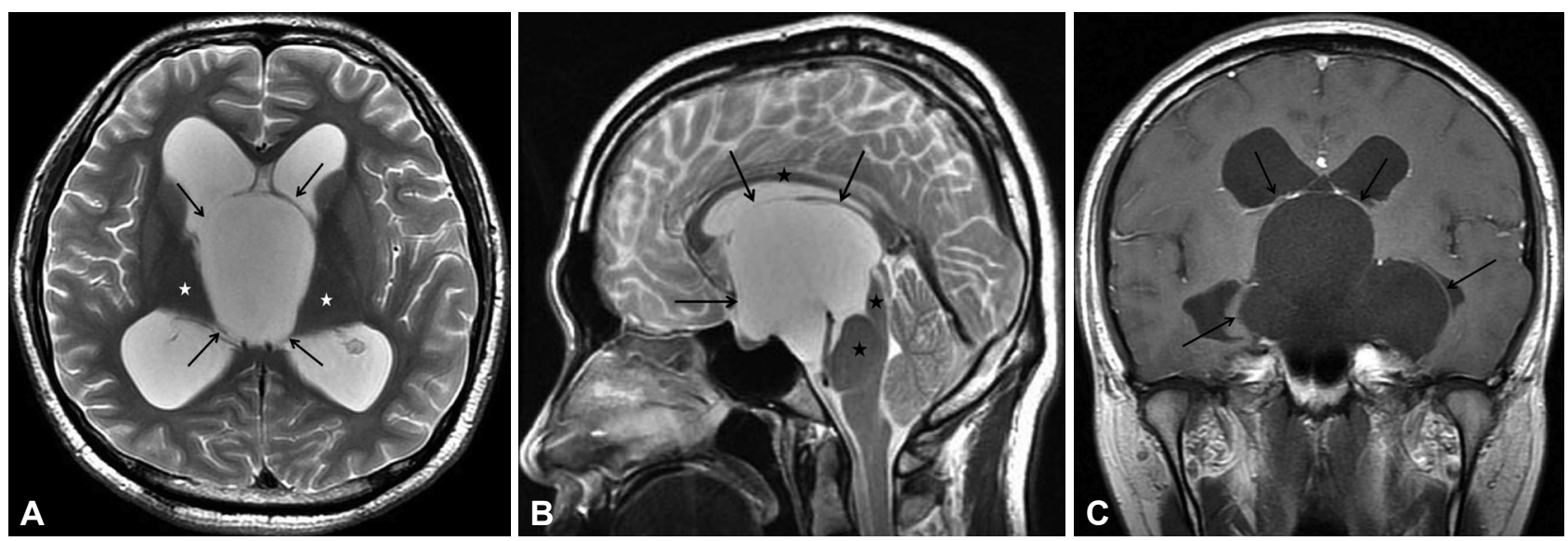

Figure 1. Axial and sagittal views of T2-weighted image (A and B) and gadolinium-enhanced T1-weighted image (C) show large suprasellar arachnoid cyst without contrast enhancement (arrows). A cyst extends into the third ventricle superiorly and compresses midbrain and pons posteriorly. Hydrocephalus due to flow disturbance in the foramen of Monro and aqueduct of Sylvius is observed. Note the compressed bilateral thalami (white stars), midbrain, pons, corpus callosum, and cingulate gyrus (black stars) and the absence of sulcal effacement or periventricular edema. 

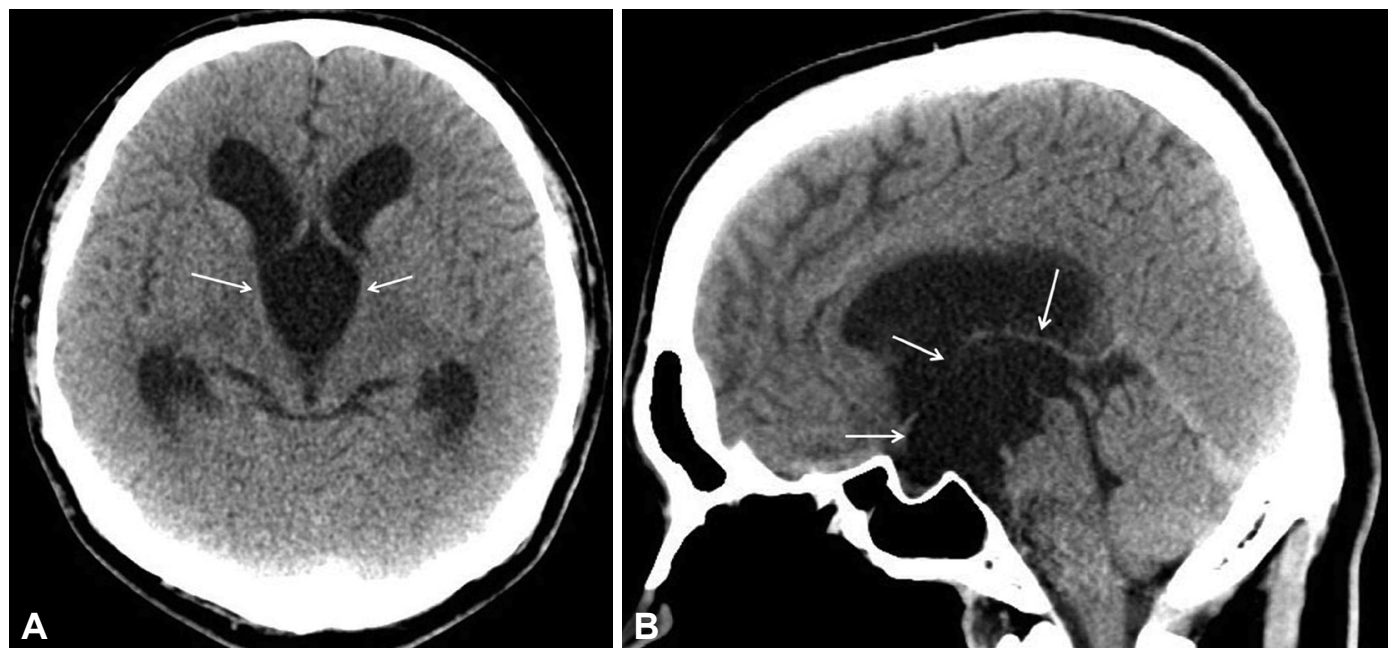

Figure 2. Postoperative computed tomographic scan after a week shows decrease in size of the cyst and restoration in shape of midbrain, pons, and corpus callosum (A and B). Note that hydrocephalus and the compressed features of bilateral thalami remain owing to decreased compliance from the adaptive changes to long-standing pressure effects.

to pressure from pulsation within the cyst on surrounding structures, such as the thalamus, basal ganglia, and motor cortex. In addition, they reported a pressure effect on the dorsomedial thalamic nucleus. Hagebeuk et al. ${ }^{8}$ reviewed and discussed 30 cases of BHDS and suggested that the red nucleus was an important site of symptom onset and aggravation. Although many hypotheses have been proposed, the exact cause of BHDS remains unclear.

Suprasellar AC is one of common causes of BHDS and accounts for about $9 \%$ of intracranial ACs. ${ }^{9}$ The majority of these lesions present as an asymptomatic mass; however, they can become symptomatic because of cyst enlargement or hemorrhage. With production of fluid from mesothelial cells of the cyst wall, the size of the AC might increase. Occasionally, one-way valve flow of CSF from subarachnoid space could increase the size of the cyst. ${ }^{10}$

Accordingly, in our presented case, we hypothesized that the recent rapid growth in the pre-existing large suprasellar $\mathrm{AC}$ was because of the relatively late onset of symptoms, atypical mixed stereotypy pattern, and suspected imaging features. On brain MRI, some evidence of adaptive changes to long-standing hydrocephalus, such as the absence of effacement in the sulcal space and inapparent periventricular edema, was observed. Before the recent growth, the pressure effect of the $\mathrm{AC}$ on the periventricular neuronal structures was under the symptomatic threshold. Because of unknown provoking factors, the pressure effect of the AC abruptly increased beyond the threshold of adjacent neuronal structures, that is, presumably, the dorsomedial thalami, basal ganglia, red nucleus, and motor cortex. Head-bobbling then began.

In addition, we should focus on the manifestation of manic symptoms unique to this case. In a recently proposed con- sensus model for mania, ventral prefrontal-limbic modulation was found to play a key role in emotional function in humans. ${ }^{11-13}$ In the context of the structural aspect, possible mechanisms of hypomanic symptoms due to the pressure effect of AC can be established. In imaging, direct pressure of the AC affected the bilateral thalami, globus pallidus, ventromedial striatum, and nucleus accumbens. Indirectly, secondary obstructive hydrocephalus transmits pressure to the corpus callosum, cingulate gyrus, and even the amygdala. ${ }^{13}$ Pressure effects to this limbic region could produce a functional alteration, and mood symptoms could develop.

Mood symptoms are the result of complex modulation or regulation of neuronal networks. ${ }^{13}$ Therefore, it probably takes time for structural influences to exert their functional influences. Moreover, mood symptoms are thought to be caused by wider structural influences. In this case, mood symptoms developed about 1-2 months after stereotypy began. After decompressive surgery of the AC, mood symptoms persisted for 1-2 months, and were managed with medication. This clinical course shows temporal correlation with onset features and supports our hypothesis. Our patient has been doing well for the past 4 years without recurrence of symptoms.

Conclusively, the long-standing hydrocephalus caused a subclinical extensive adaptive alteration in neural structures including the limbic system. Recent growth of the cyst may have triggered an unusually wide range of symptoms. We expect our case may provide some clues to structurally understand the pathology of bipolar disorder.

In summary, we report our successful experience of surgically treating stereotypy and hypomania developed in the rare BHDS. We suggest that there are surgically treatable stereotypies frequently encountered in clinical practice. Fur- 
thermore, if stereotypies do not respond to appropriate treatment and other symptoms are complicated, brain imaging might be essential.

\section{REFERENCES}

1. Bhattacharyya KB, Senapati A, Basu S, Bhattacharya S, Ghosh S. Bobble-head doll syndrome: some atypical features with a new lesion and review of the literature. Acta Neurol Scand 2003;108:216-220.

2. Russo RH, Crosby EC, Kindt GW. The bobble-head doll syndrome: experimental study of anatomic basis. Surg Forum 1974;25:466-468.

3. Ahn Y, Cho BK, Wang KC. Bobble-head doll syndrome associated with subduroperitoneal shunt malfunction. Childs Nerv Syst 1997;13:234237.

4. Ramesh S, Raju S. Suprasellar arachnoid cyst presenting with bobblehead doll syndrome: report of three cases. J Pediatr Neurosci 2015;10: 18-21.

5. Benton JW NG, Huttenlocher PR, OjemannRG, Dodge PR. The bobblehead doll syndrome. Report of a unique truncal tremor associated with third ventricular cyst and hydrocephalus in children. J Neurol 1966;16: 725-729.

6. Ishihara M, Nonaka M, Oshida N, Hamada Y, Nakajima S, Yamasaki M. "No-no" type bobble-head doll syndrome in an infant with an arach- noid cyst of the posterior fossa: a case report. Pediatr Neurol 2013;49: 474-476.

7. Benton JW NG, Huttenlocher PR, Ojemann RG, Dodge PR. The bobblehead doll syndrome: report of a unique truncal tremor associated with third ventricular cyst and hydrocephalus in children. J Neurol 1966;16:725-729.

8. Hagebeuk EE, Kloet A, Grotenhuis JA, Peeters EA. Bobble-head doll syndrome successfully treated with an endoscopic ventriculocystocisternostomy. Case report and review of the literature. J Neurosurg 2005; 103(3 Suppl):253-259.

9. Desai KI, Nadkarni TD, Muzumdar D, Goel A. Suprasellar arachnoid cyst presenting with bobble-head doll movements: a report of 3 cases. Neurol India 2003;51:407-409.

10. Eustace S, Toland J, Stack J. CT and MRI of arachnoid cyst with complicating intracystic and subdural haemorrhage. J Comput Assist Tomogr 1992;16:995-997.

11. Townsend J, Altshuler LL. Emotion processing and regulation in bipolar disorder: a review. Bipolar Disord 2012;14:326-339.

12. Blond BN, Fredericks CA, Blumberg HP. Functional neuroanatomy of bipolar disorder: structure, function, and connectivity in an amygdalaanterior paralimbic neural system. Bipolar Disord 2012;14:340-355.

13. Strakowski SM, Adler CM, Almeida J, Altshuler LL, Blumberg HP, Chang KD, et al. The functional neuroanatomy of bipolar disorder: a consensus model. Bipolar Disord 2012;14:313-325. 